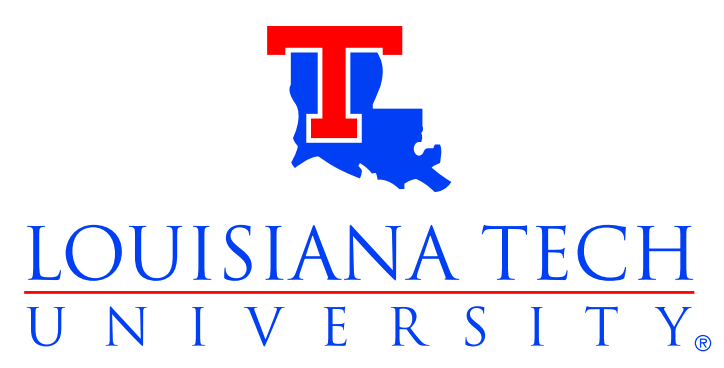

\title{
FINAL REPORT: High Energy Physics Research at Louisiana Tech
}

From the

Louisiana Tech University

Center for Applied Physics Studies

Submitted June 28, 2013

Institution:

Street Address:

City/State/ZIP:

Principal Investigator

Postal Address:

Telephone Number:

Email Address:

DOE Grant Number:

Period Covered:

DOE/Office of Science Program Office:

DOE/Office of Science Program Office Technical Contact:

Co-principal Investigators:
Louisiana Tech University

1 Adams Blvd

Ruston, LA 71272

Dr. Lee Sawyer

P.O.Box 10348, Ruston, LA

318-257-4053

sawyer@phys.latech.edu

DE-FG02-99ER41117

June 19, 1999 to April 30, 2013

Office of High Energy Physics

Dr. Simona Rolli

simona.rolli@science.doe.gov

Dr. Zeno D. Greenwood

greenw@phys.latech.edu

Dr. Markus Wobisch

wobisch@latech.edu 


\section{Introduction}

Since June, 1999, the Louisiana Tech University High Energy Physics group has received base funding from Dept.of Energy grant DE-FG02-99ER41117, with periodic renewals, supplemented by matching state and university funds, and grants for computing and detector development. This funding, which amounted to a total of $\$ 2,843,350$, has facilitated the establishment of a High Energy Physics research group Louisiana Tech University, led to major involvement with the upgraded $D \varnothing$ experiment at the Fermilab Tevatron collider and the ATLAS experiment at the CERN Large Hadron Collider, contributed to the growth of the Louisiana Tech physics program from a terminal Masters program to a PhD-granting program, supported several Masters and $\mathrm{PhD}$ students in the completion of their graduate research work, and underwritten several postdoctoral researchers in the field.

The Louisiana Tech University High Energy Physics group is a medium-sized experimental particle physics research group with faculty, postdoctoral researchers, doctoral and master's students, and undergraduate students engaged in operations and data analyses at hadron colliderbased experiments. After several years of collaborating on the $D \emptyset$ experiment at the Tevatron $p \bar{p}$ collider at Fermi National Accelerator Laboratory (Fermilab), our primary focus is now centered on the ATLAS experiment at the LHC $p p$ collider at the CERN laboratory near Geneva, Switzerland.

The group consists of three faculty members: Dr. Lee Sawyer (Charles \& Newllyn Spruell Professor of Physics and Director of Chemistry \& Physics at Louisiana Tech, and member of the DØ and ATLAS experiments), Dr. Zeno D. "Dick" Greenwood (W. W. Chew Professor of Physics and Louisiana Tech team leader on ATLAS), and Dr. Markus Wobisch (Eva J. Cunningham Associate Professor of Physics, member of the D $\varnothing$ and ATLAS experiments, and coordinator of the FASTNLO project). We have had eight postdoctoral researchers (one current) who have made significant contributions to many aspects of the D $\varnothing$ and ATLAS experiments. We have had several students in the Computational Analysis and Modeling PhD program who have worked with our group, including one current student on ATLAS. In 2007, Louisiana Tech was granted approval to add an Engineering Physics PhD track to its interdisciplinary PhD program ${ }^{1}$. Since then, we have had five doctoral students join our group. The first student to finish his doctoral degree on ATLAS, Ram Dhullipudi, graduated in summer, 2012. Indeed, the High Energy Physics group and its successes in securing funding and producing quality physics results were specifically noted by the Board of Regents in the creation of the Engineering Physics PhD track. In the past year, we also had one CAM PhD student, Scott Atkins, and one Engineering Physics PhD students, Kiran Chakravrathula, who completed their dissertations on the $\mathrm{D} \emptyset$ experiment. We also had one Masters student, Alex Johnson, who wrote his thesis on an ATLAS analysis.

With the end of collider operations at the Tevatron, our group is in the process of winding down our work on DØ. This summer our last doctoral students on D $\emptyset$ graduated. We have recently published two papers on jet physics analyses on $D \emptyset$, with two more near submission and another one or two papers to be written later this year. Our focus is now primarily on the ATLAS experiment at the LHC. We have established important service roles in two major areas:

\footnotetext{
${ }^{1}$ As a research university, Louisiana Tech's "niche" is in interdisciplinary science and engineering research and education. All graduate programs in our College of Engineering and Science are interdisciplinary by design. The Engineering Physics PhD is administered by the Physics Program, which sets curriculum, sets qualifying examinations, etc.
} 
Triggers and Data Quality. These will discussed in detail below.

During the past three years, we have had two excellent postdocs, Dr. Catrin Bernius and Dr. Matthew Tamsett, with extensive previous experience on ATLAS, who have significantly extended the impact of our group. Our physics research is also focused in two areas: Top quark physics in final states containing tau leptons, and studies of the strong interaction in jet production. Louisiana Tech has developed a reputation as one of the leading institutions pursuing jet physics studies at the Tevatron, and we are building a similar reputation on ATLAS, with several jet analyses in progress. Focusing on closely related topics in the jet and top analyses and coordinating these analyses in our group between $D \emptyset$ and ATLAS has led to high efficiency and increased visibility inside the individual collaborations and beyond.

The goal of this project was to create, maintain, and strengthen a world-class, nationally and internationally recognized experimental high energy physics group at Louisiana Tech University, focusing on research at the energy frontier of collider-based particle physics, first on the $D \varnothing$ experiment and then with the ATLAS experiment, and providing leadership within the US high energy physics community in the areas of jet physics, top quark and charged Higgs decays involving tau leptons, as well as developing leadership in high performance computing.

\section{Group Description and Activities}

\subsection{Group Description}

The Louisiana Tech High Energy Physics research group in 2012 consisted of three physics faculty members; two postdoctoral researchers; three Engineering Physics PhD candidates; one Computer Analysis and Modeling (CAM) PhD candidate; and three undergraduate students. Our support staff includes a computer system manager, a technician and a secretary; all shared with other groups in the Center for Applied Physics Studies. In the past year we graduated two Engineering Physics PhDs (one on D $\varnothing$ and one on ATLAS), two CAM PhDs (one on D $\varnothing$ and one who worked on High Availability Computing in conjunction with ATLAS), and one masters' (on ATLAS).

The Louisiana Tech group was formed by Sawyer in 1997. We were initially involved exclusively with the $\mathrm{D} \emptyset$ experiment. After the group grew with the addition of Greenwood in 1999 and Wobisch in 2007, we joined ATLAS in 2006 as a sub-institute of the Brookhaven National Laboratory institute. In July 2012, with the support of the US-ATLAS management, the Louisiana Tech group formally applied for full institutional membership, based on our complement of personnel and high level of activity on the experiment. This application was approved at the ATLAS collaboration meeting in February 2013.

\subsection{Group Activities}

The Louisiana Tech group has focused it efforts in a few areas in which it can be successful in producing high quality physics results, providing service to the experiments, and training graduate students. We work as a very closely integrated team; often, for example, with one faculty member supervising students' service work while another faculty member supervises their physics analyses. In this section, we provide an overview of these efforts, with details of particular analyses described in the sections of the appropriate principal investigators. Major 
group activities also include the training of incoming students and the professional development of students and postdocs. This has lead to the creation of a specialized graduate class on hadron collider physics by Wobisch with close one-on-one interactions with the students.

\section{The Louisiana Tech Jet Physics Analysis Team}

Principal investigators Sawyer and Wobisch, postdoc Tamsett, students Subramaniam and Alghadeer, along with recent graduates Dhullipudi, Atkins, Chakravarthula, and Johnson form the Louisiana Tech Jet Physics Analysis Team. The team members work seamlessly together on a variety of closely related multijet analyses simultaneously on the D $\varnothing$ and ATLAS experiments, with tasks shared seamlessly. Between June 2009 and March 2013, we have published the results of seven D $\varnothing$ and two ATLAS jet analyses, and one phenomenology paper. Therefore, it is not excessive to say that we have become the leading jet group at the Tevatron, and we are developing a strong reputation in jet physics on ATLAS. The main focus of the Jet Analysis Team are studies of multijet production rates and their sensitivity to New Physics phenomena, parton distribution functions, and the strong coupling constant $\alpha_{s}$. Our measurement and subsequent phenomenological analysis of dijet angular distributions on $D \emptyset[1]$ have provided the most stringent limits for New Physics models assuming quark compositeness or spatial extra dimensions, prior to the LHC turn-on. Measurements of the production cross sections of dijet and three-jet final states as a function of dijet and three-jet invariant mass have provided additional constraints on parton distribution functions $[2,3]$.

Most of our current analysis efforts are focused one of the fundamental parameters in the standard model, the strong coupling constant $\alpha_{s}$, and its running as a function of energy. A determination of $\alpha_{s}$ from the $\mathrm{D} \emptyset$ measurement of the inclusive jet cross section [4] has provided the most precise $\alpha_{s}\left(M_{Z}\right)$ result from a hadron collider. As detailed in our recent publication [5], determinations of $\alpha_{s}$ at highest energies are preferably based on variables which are independent (or depend only weakly) on the parton distribution functions, in order to avoid circular arguments. Therefore, our determinations of $\alpha_{s}$ are based on variables which are ratios of multijet cross sections, in which parton distributions functions cancel to a large extent. With this approach we extract $\alpha_{s}$ up to the largest $p_{T}$ accessible at the Tevatron and the LHC. We have study different quantities, some of which we have invented for this purpose [6]. The quantities studied are $R_{3 / 2}$ (the ratio of inclusive three-jet and dijet cross sections), $R_{\Delta R}$ (the average number of neighboring jets by which jets are accompanied) $R_{\Delta \phi}$ (the fraction of dijets with a given azimuthal decorrelation), and a set of nine event shape variables. Measurements of these quantities have been published $[5,7,8]$ in the $\mathrm{D} \emptyset$ experiment. The corresponding measurements on ATLAS are currently in progress, and the first results will be published in 2013 and in 2014. Figure 1 shows results of the three recent measurements of $R_{\Delta R}, R_{3 / 2}$, and $R_{\Delta \phi}$ on D $\emptyset$, and the $\alpha_{s}$ results extracted from the $R_{\Delta R}$ measurement.

\section{The Louisiana Tech Top Physics Analysis Team}

The Louisiana Tech Top Physics Analysis Team is comprised of Principal Investigator Greenwood, Postdoc Bernius, and student Sircar. Greenwood has been convener of the ATLAS ttbar-to-tau working group since its inception in 2010, and with Bernius and Sircar, have been actively investigating Top physics analyses involving $\tau+X$ final states. The group first concentrated on 

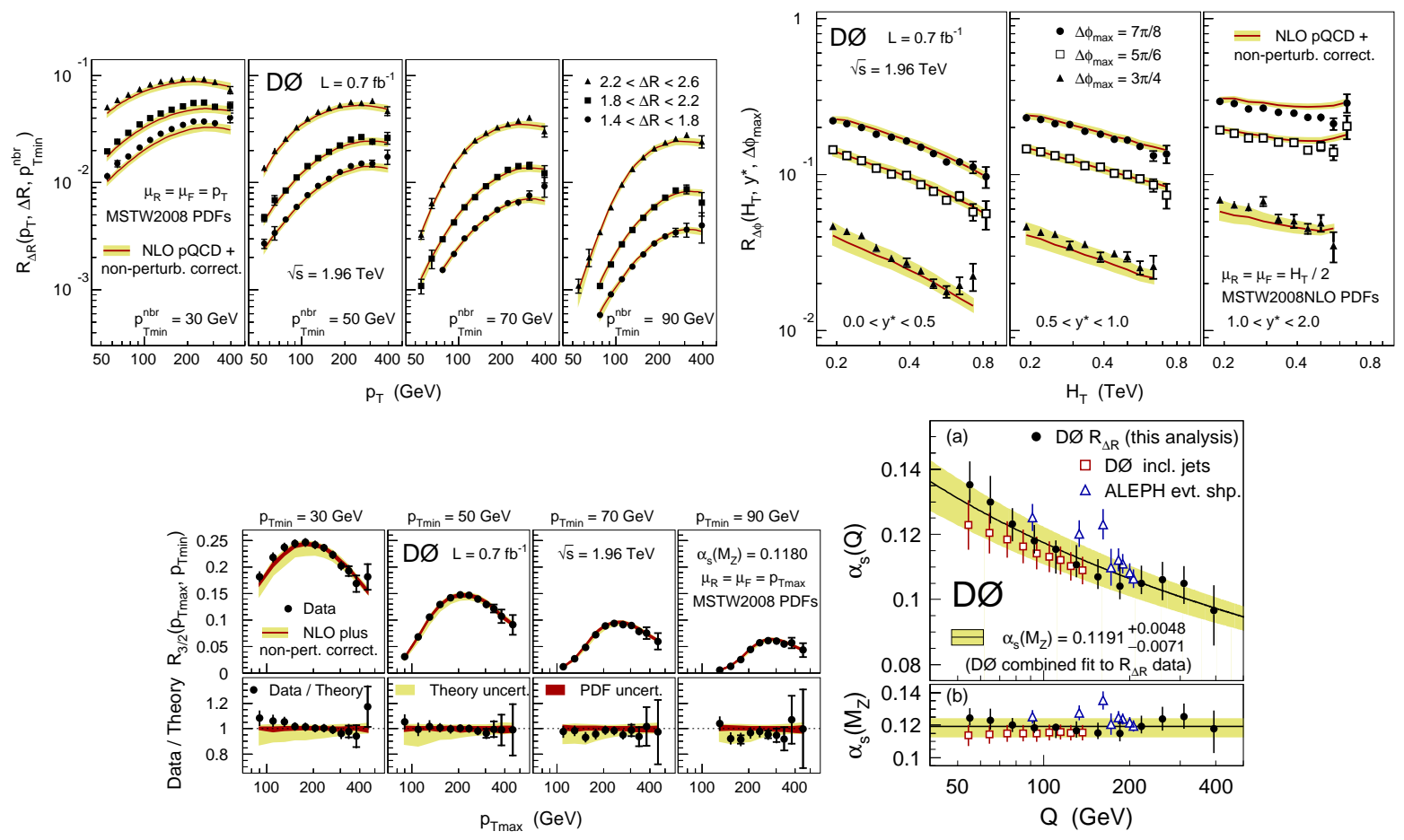

Figure 1: Measurements of multijet cross section ratios in the D $\emptyset$ experiment $R_{\Delta R}$ (top left), $R_{\Delta \phi}$ (top right), and $R_{3 / 2}$ (bottom left), and the extracted $\alpha_{s}$ result (bottom right).

measuring the top quark pair $(t \bar{t})$ production cross section in the $l+\tau$ channel. Greenwood and Bernius were analyzers on this project, and Sircar contributed to the development of the Boosted Decision Tree tools used for the tau identification. This work resulted in supporting conference notes and a publication Ref. [9]. In parallel, Greenwood and Bernius were involved in a search for light charged Higgs bosons in the $\tau+$ jets final state in $t \bar{t}$ decays. A major contribution to the analysis was the determination of the extensive QCD background employing the ABCD method. This work also resulted in a publication [10], for which Bernius was co-editor of an internal note [11].

The expertise gained in our light charged Higgs search has been applied in top quark pair production cross section measurement analyses in the $\tau+$ jets channel with 2011 and 2012 data, since the same final states are involved. Greenwood and Sircar worked on the 2011 analysis, which will result in a publication and a thesis for Sircar. Greenwood and Bernius are currently working on the 2012 analysis.

\section{Senior Investigator Zeno D. (Dick) Greenwood}

Postdoc: Dr. Catrin Bernius

PhD students: Anirvan Sircar, David Palma

Computer Engineer Michael Bryant

Greenwood has been Team Leader for the Louisiana Tech ATLAS group since the group joined 
ATLAS in 2006. His Team Leader duties include representing the group on US ATLAS and ATLAS institutional matters, which include updating the group membership databases (US ATLAS and the ATLAS "At a Glance" databases), overseeing the group ATLAS Team Account, and working with detector and trigger groups to establish authorship service tasks for Louisiana Tech group members. Greenwood oversees the work of Postdoc Catrin Bernius who joined the group in October 2010, and doctoral student Anirvan Sircar, who was stationed at CERN during 2010-2011. Greenwood also directs the work of Computer Engineer Michael Bryant and Graduate student David Palma in the design, installation and maintenance of our Tier 3 and grid computing system.

\subsection{ATLAS Trigger}

Bernius has been a member of the trigger menu group since December 2010. Her work can be divided into two areas, the implementation of trigger items in the trigger software and active involvement in trigger operation as trigger expert on-call.

The implementation and (re-)configuration of trigger items is done in two steps, the chain definition for each trigger level with respect to thresholds and algorithms. Trigger chains as well as menus in general are continually evolving due to changing run conditions as well as the feedback and requests from physics and performance groups. This work provides great insight into the vast physics analyses in the ATLAS collaboration.

Bernius' involvement in trigger operation is in the form of $24 / 7$ trigger menu oversight as well as online expert on-call shifts. These on-call shifts require close interaction with trigger operation, trigger menu coordination and run coordination as well as close contact with the trigger shifter. Further tasks include the monitoring of physics data-taking, reacting to any trigger-related problems during operation and taking appropriate action, and assisting detectors with trigger setups for special runs and tests.

\subsection{Research: Top and Higgs Physics in $\tau+X$ Final States}

\section{Hadronic tau Identification using Boosted Decision Trees}

Hadronically decaying $\tau$ leptons are reconstructed either from calorimeter or track seeds, where quality cuts are applied on the reconstructed jets and tracks respectively. This way of reconstruction provides very little rejection against QCD jet backgrounds. Although the backgrounds from electron production are significantly lower in rate, they are very difficult to distinguish from the tau decays with one charged track (1-prong decay). The rejection comes from a separate identification step and is usually based on several discriminating variables. These methods include selections based on simple cuts, projective likelihood variables, and Boosted Decision Trees (BDTs). The input to the BDT are discriminating variables which distinguish $\tau$ jets from quark and gluon jets as well as from electrons. General properties useful for distinguishing a $\tau$ jet from quark and gluon jets include particle multiplicity and the width of a jet. While an electron may also be distinguished from a $\tau$ through the transverse shower shape, the longitudinal development plays a stronger role than in the jet-rejection case.

Sircar worked on improvements of the electron BDT in support of the TauID group and specifically for the ttbar to tau + lepton analysis. This work is a technique to remove electrons 

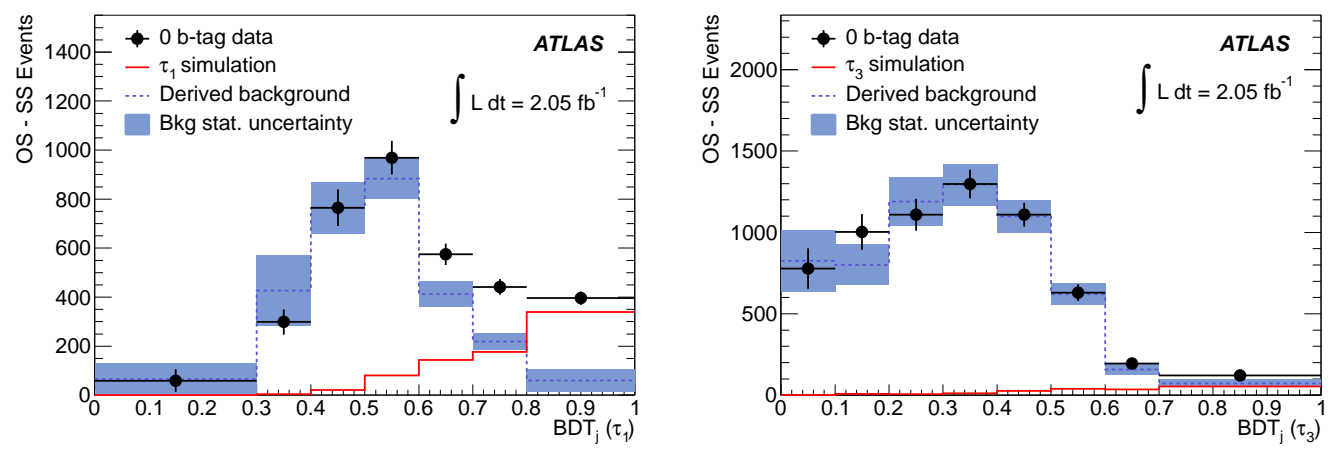

Figure 2: BDTj (OS-SS) distributions of lepton + tau (e and $\mu$ combined) events in the 0 b-tag data (black points). The plots on the left are 1-prong taus and on the right, 3-prong taus. The shapes of these background templates are used for the fits to the $\geq 1 \mathrm{~b}$-tag distributions after applying MC corrections.

faking 1-prong taus. Sircar's contributions include setting up the initial machinery for training the BDT and choosing a set of discriminating variables for the initial training.

\section{Top quark pair production cross section in the $l+\tau$ channel}

Measuring the top quark pair $(t \bar{t})$ production cross section $\sigma(t \bar{t})$ using a channel with $\tau$ leptons is of great interest because it can open a window to physics beyond the Standard Model (SM) (see for example, the Charged Higgs search discussed below). In the SM, the top quark decays nearly $100 \%$ of the time into a $\mathrm{W}$ boson and a $b$-quark, and $t \bar{t}$ pairs are identified by either the hadronic decays ( $W \rightarrow q \bar{q})$ or leptonic decays $(W \rightarrow l \nu)$ of the $\mathrm{W}$ boson and the presence of additional jets.

Our analysis used $2.05 \mathrm{fb}^{-1}$ collected by ATLAS at $\sqrt{s}=7 \mathrm{TeV}$ between March and August 2011. After application of kinematic selection criteria that require one top quark to decay via $W \rightarrow l \nu$ (where $l$ is either a muon or an electron) and identification of a jet as originating from a b quark (b-tag), the dominant background to the $t \bar{t} \rightarrow l+\tau+X$ channels with the lepton decaying hadronically is the $t \bar{t} \rightarrow l+$ jets channel in which a jet is misidentified as a hadronic $\tau$ lepton decay. Therefore, $\tau$ lepton identification ( $\tau$ ID) is critical for separating signal and background. The $\tau$ ID methodology employed in this analysis exploits a boosted decision tree (BDT) algorithm. The number of $\tau$ leptons in a sample is extracted by fitting the distributions of BDT outputs to background and signal templates, as shown in Fig. 2 for 1-prong and 3-prong tau candidates. The results were checked using an alternative matrix method, based on a cut on the BDT output. This cross section measurement has been published in Ref. [9].

\section{$H^{ \pm}$bosons in the $\tau+$ jets final state in $t \bar{t}$ decays}

Greenwood and Bernius have been involved in the light charged Higgs boson via the apparent violation of lepton universality in top quark decays analysis in 2011/2012. The main contribution was the estimation of background arising from QCD processes from data, using the ABCD method. The ABCD method defines four regions in two uncorrelated variables, and works under the assumption that the regions have similar ratio over each other for those background com- 
ponents of each region in the data. The signal is located in one of the four regions. It is then possible to estimate the number of background events in the signal region. Bernius has been co-editor of the internal note for the analysis of the $H^{+} \rightarrow \tau+$ jets final state [11] which evolved to a publication [10]. Bernius is currently the trigger contact for the $\tau+$ jets final states analyses.

\section{Top quark pair production cross section in the $\tau+$ jets channel with 2011 and 2012 data}

So far, no published measurement of the top pair production cross section in the channel $t \bar{t} \rightarrow$ $\tau \nu b j j b$ with $\sqrt{s}=8 \mathrm{TeV}$ data has been conducted at the LHC. This study would provide an important alternative measurement of the top pair cross section at higher energies. Also, such a measurement would demonstrate that the signature of hadronically decaying $\tau$ leptons is well understood at the ATLAS experiment, even in the busy environment of a $t \bar{t}$ event with increased number of pile-up events compared to the $2011 \sqrt{s}=7 \mathrm{TeV}$ data.

The $t \bar{t}$ channel has the same final state as the charged Higgs search analysis to which the Louisiana Tech group (Greenwood and Bernius) contributed. The expertise gained in the $H^{ \pm}$ analysis will help to achieve a cross-section measurement in the channel $t \bar{t} \rightarrow \tau+$ jets.

\subsection{Computing}

The ATLAS Tier 3 Computing Center at Louisiana Tech is distinctive for its computing and storage resources, active involvement in grid computing, and its high-speed connectivity to powerful computing clusters within the state. Our Tier 3 infrastructure, outlined below, consists of both local and remote resources and gives us the capacity and expandability needed for storing large data sets. The connection to the Open Science Grid (OSG) enables our Tier 3 resources to be accessible on the grid for remote job and data management. By leveraging our state's advanced optical network, we have the local capabilities required for processing, storing, and analyzing ATLAS data at our site.

The Tier 3 center is supported by Greenwood, a full-time systems manager (Bryant), and student personnel (primarily D. Palma) who aid in the day-to-day operations of the cluster. We have also participated strongly in the Open Science Grid since 2006 and are a key member in the Distributed Organization for Scientific and Academic Research (DOSAR). Furthermore, Greenwood has played a prominent role in remote computing on the D $\varnothing$ Experiment and in the grid computing community as convener of DOSAR.

In mid-2010, we were awarded DOE ARRA supplemental funds to build a new, robust Tier 3 center at Louisiana Tech. The cluster initially consisted of 13 compute nodes (140 cores/280 threads) for our LaHEP group and two physics/engineering groups that supplied additional funding. As part of a shared computing campus initiative, an expansion earlier this year by the Chemistry and Computer Science departments added another 12 compute nodes (768 cores) to the community cluster. Many parallel processing systems are available to researchers on the cluster, including PROOF on Demand, Hadoop's Map/Reduce framework, GNU Parallel, and standard MPI/OpenMP libraries. As designed, our LaHEP researchers can run local batch jobs on the Tier 3 and LONI resources, submit grid jobs to both sites through the OSG compute elements, and transfer data into and out of the robust Hadoop storage system at our site using the OSG storage element. 
Other advances in the last year include local ATLAS analysis and continued D $\varnothing$ Monte Carlo production. We have been actively using the Tier 3 and LONI clusters for analyzing and processing ATLAS data stored in the Hadoop file system. Through our OSG compute element at LSU, we have been running D $\emptyset$ simulations on one of the LONI 5 TF clusters (Eric). We have completed 11.2 million events during the past year and over 52 million events total.

\section{Senior Investigator Markus Wobisch}

Postdoc: Dr. Matthew Tamsett

PhD student: Rajivalochan Subramaniam

PhD students finished in 2012: Dr. Scott Atkins, Dr. Kiran Chakravarthula

Masters Student finished in 2012: Alex Johnson

In the physics community Wobisch is well known for his work at HERA and at the Tevatron and his expertise on QCD and jet physics. He has made significant and innovative contributions through experimental measurements of jet production in deep-inelastic scattering and in hadron-hadron collisions as well as through phenomenological studies and interpretations of these measurements. The main focus of Wobisch's current work are studies of jet production rates, and their phenomenological interpretation in searches for New Physics phenomena, determinations of parton distribution functions (PDFs) of the proton, and extractions of the strong coupling constant $\alpha_{s}$. The work comprises experimental analyses in the $\mathrm{D} \varnothing$ experiment at the Fermilab Tevatron Collider and in the ATLAS experiment at the CERN LHC, as well as technical service work on the ATLAS jet trigger and on the $\mathrm{D} \emptyset$ jet energy calibration. In addition, Wobisch is active in phenomenology as part of the experimental analyses, and also as the leader of the fastNLO project.

Closely related to the research program is the service work. In the previous years, Wobisch and Atkins have contributed to the $\mathrm{D} \emptyset$ jet energy calibration. Wobisch, Tamsett, and Subramaniam are all working on the ATLAS jet trigger, and Tamsett served as co-convener of the ATLAS jet trigger group.

\subsection{Jet Physics on ATLAS and D $\emptyset$}

During the last years, Wobisch, in collaboration with Sawyer, has established the Louisiana Tech group as an innovative and creative force, leading the Run II Tevatron jet physics program. Between 2007 and 2008, Wobisch had developed a fast parametrized simulation of the DØ detector for jet measurements (DØJetSim) in which he implemented all relevant detector resolutions and efficiencies, and the corresponding uncertainties. As a flexible tool, DØJetSim has built the basis for the strong track record of the Louisiana Tech group in the $D \emptyset$ experiment. This has resulted in a comprehensive set of seven $D \emptyset$ multijet measurements which have been published during 2009-2013. Four of these measurements are performed simultaneously in the ATLAS experiment, two of which are aimed to be published before summer $2014^{2}$. The published measurements have already had a strong impact, as they resulted in the best pre-LHC

\footnotetext{
${ }^{2}$ This has also resulted in three theses: Two doctoral dissertations in DØ and one masters' thesis in ATLAS.
} 
limits on three New Physics models, the most precise $\alpha_{s}$ result at a hadron collider, and in the invention of new multijet variables, some of which are already adopted by ATLAS and CMS.

Dijet Angular Distributions in $\mathbf{D} \emptyset$ The dijet angular distributions in hadron collisions are sensitive to the low energy tails of high-energy phenomena. For the first time, Wobisch (in collaboration with N. Parua at Indiana University) has measured dijet angular distributions [1] in Run II, with searches for quark compositeness, and extra spatial dimensions (in the ADD Large Extra Dimensions, and the $\mathrm{TeV}^{-1}$ Extra Dimensions models). The published limits [1] are the best corresponding limits in the pre-LHC area, and were featured in the 2010 PDG Review of particle physics [12].

Dijet and Three-Jet Mass Cross Sections in DØ Multijet differential cross sections as a function of the multijet invariant mass are classical observables to test QCD dynamics, and to probe the PDFs of the proton. Wobisch (in collaboration with M. Rominsky and M. Strauss at Oklahoma University, and D. Lincoln at Fermilab) has measured for the first time the rapidity dependence of the dijet invariant mass distribution, published by $D \varnothing$ in Ref. [2] (the same approach has since also been adopted by both LHC experiments), and (together with Z. Hubacek at Prague University), the rapidity and $p_{T}$ dependence of the three-jet invariant mass distribution in $\mathrm{D} \emptyset[3]$.

Measurement of Angular Correlations of Jets, $\boldsymbol{R}_{\boldsymbol{\Delta}}$, in D $\emptyset$ and ATLAS During 2010, Wobisch had invented and studied a new variable, $R_{\Delta R}$, which probes the angular correlations of jets in the plane of rapidity $y$ and azimuthal angle $\phi$. The variable is defined as the average number of neighboring jets that accompany a given jet (from an inclusive jet sample). In collaboration with Sawyer, Wobisch has measured $R_{\Delta R}$ on $\mathrm{D} \emptyset$, and the results are published in Phys. Lett. B [5] (see also Fig 1 top left). Based on these measurements, the strong coupling $\alpha_{s}$ was extracted (see below). Currently, Wobisch and Subramaniam are measuring $R_{\Delta R}$ also on ATLAS (together Tamsett and Sawyer). An initial study, based on the ATLAS data at $\sqrt{s}=7 \mathrm{TeV}$, is now extended to the 2012 data at $\sqrt{s}=8 \mathrm{TeV}$. First results have been presented in internal ATLAS meetings and the measurement will be published by summer 2014 .

Measurement of $\boldsymbol{R}_{\mathbf{3} / \mathbf{2}}$ in D $\emptyset$ and ATLAS An ATLAS measurement of $R_{3 / 2}$ was published in September 2011 [13], to which Tamsett contributed as a member of the analysis team and as the editor of the internal supporting document. A measurement of $R_{3 / 2}$ has also been performed using $\mathrm{D} \emptyset$ data, by Sawyer (data analysis and editor of analysis note and paper draft), Atkins (control plots for MC and data comparison), Wobisch (MC simulation, data correction, and theory calculations), and published in Phys. Lett. B [7] (see also Fig 1 bottom left).

Rapidity and $p_{T}$ Dependence of Dijet Azimuthal Decorrelations in D $\emptyset$ and ATLAS In a recent publication, the LA Tech group has proposed the new quantity, $R_{\Delta \phi}$, for studying the rapidity dependence of dijet azimuthal decorrelations at hadron colliders [6]. The measurement of $R_{\Delta \phi}$ using $\mathrm{D} \emptyset$ data was Chakravarthula's doctoral dissertation topic, supervised by Wobisch. The results have been published in Phys. Lett. B [6]. At the same time, the $R_{\Delta \phi}$ measurement is also performed using ATLAS data at $\sqrt{s}=8 \mathrm{TeV}$ by Tamsett, Sawyer, Wobisch, in collaboration 
with M. Begel (BNL) as part of Wobisch's EPSCoR grant. The results are currently in review by the ATLAS editorial board and expected to be published during fall 2013.

Measurement of Event Shapes in D $\emptyset$ and ATLAS Event shape variables have been popular in $e^{+} e^{-}$collisions for testing pQCD and for determinations of $\alpha_{s}$. Recently, the CDF and the CMS collaborations have published first measurements of a limited set of event shapes at hadron colliders.

Atkins (supervised by Sawyer and Wobisch) has just finished his doctoral thesis, with a measurement of nice event shape variables in $\mathrm{D} \emptyset$. The same event shape variables were also measured using ATLAS data at $\sqrt{s}=7 \mathrm{TeV}$ as Johnson's Masters Thesis (supervised by Wobisch). Our group will approach a full ATLAS measurement of these event shapes during 2014.

Determinations of $\boldsymbol{\alpha}_{s}$ in a new Energy Regime in D $\emptyset$ and ATLAS In 2009, Wobisch has used the $\mathrm{D} \emptyset$ inclusive jet data to extract $\alpha_{s}$ over an energy range from $50 \mathrm{GeV}$ to $140 \mathrm{GeV}$, published by $\mathrm{D} \emptyset$ in [4]. Based on the new measurement of $R_{\Delta R}$ (discussed above), Wobisch has, again, extracted $\alpha_{s}$ results. These new results cover a much larger energy range of $50-400 \mathrm{GeV}$. This is the first result that determined $\alpha_{s}$ in an energy regime above the LEP limit of $208 \mathrm{GeV}$, and the first result from a single experiment that demonstrates the running of $\alpha_{s}$ over a factor of eight in energy. (see also Fig 1 bottom right). The forthcoming publication of the ATLAS measurements of $R_{\Delta \phi}$ will also include a determination of $\alpha_{s}$, expanding the energy range of the most recent $\alpha_{s}$ results from CMS.

\subsection{Service Work on the ATLAS Jet Trigger}

Over the past two years the contributions by Wobisch, Sawyer, Tamsett, and Subramaniam have become a vital part of the ATLAS jet trigger group activities, both in the development of new methods and the leadership of the group.

In 2011 the group developed a new L2 jet trigger algorithm termed the "L1.5" jet trigger. The L1.5 jet trigger is a new style L2 trigger algorithm which uses the "Trigger Towers" (TT) and "Jet Elements" (JE) produced at L1 to reconstruct jets across the entire detector. By studying the L1 calorimeter data with the L2 software layer L1.5 is able to provide several key enhancements to the jet trigger functionality. The use of the anti- $\mathrm{k}_{T}$ jet algorithm at L1.5 has been demonstrated to significantly improve the angular resolution for all jets and to increase the jet finding efficiency in multijet topologies by up to 20\%. The L1.5 jet trigger was successfully tested in 2011 and it was included in the trigger menu for the remainder of the 2011 lead-lead program. Further work by Subramaniam to improve the L1.5 trigger continued throughout 2012. This included the addition of calibrations to the appropriate hadronic response scale which serve to improve the energy resolution and hence utility of the system. Results from L1.5 have been presented at the LHCC, PM2012 (by Tamsett), CALOR and ICHEP conferences. In addition to this the Louisiana Tech group has become involved with the direction and leadership of this ATLAS jet trigger group. Tamsett is coordinating the writing of a paper of the performance of the jet trigger in 2011. In October 2012 Tamsett became the jet trigger convener, as a direct result of the involvement of the Louisiana Tech group in the activities listed above. As a result the 
Louisiana Tech group has become a key group responsible for the upkeep and development of the ATLAS jet trigger over the coming years.

\subsection{The fastNLO Project}

Wobisch started the fastNLO project $[14,15]$ in 2005, together with collaborators T. Kluge (University of Liverpool) and K. Rabbertz (University of Karlsruhe), designed to provide very fast calculations of cross sections in hadron-induced processes. FastNLO is currently being used in the most recent global PDF analyzes, by the MSTW group (MSTW2008 $[16,17]$ ), the CTEQ group (CT10 [18] ) and also in the PDF analyses in Refs. [19, 20, 21]. The fastNLO team has also provided a world summary figure on inclusive jet production at different colliders for the PDG books in 2010 and 2012. In 2011, two collaborators, Daniel Britzger (MPI Munich) and Fred Stober (Karlsruhe University) joined the fastNLO project. Since then the fastNLO project has published version 2 of the code [22], which is a major update, with more flexibility in the data structures, and with additional features. The new data structures allow to implement calculations for different processes (e.g. diffractive jet production, which is used at HERA to determine the pomeron PDFs).

\section{Senior Investigator H. Lee Sawyer}

PhD Student: Khadeejah Alghadeer

PhD Students finished in 2012: Scott Atkins (co-adviser), Ram Dhullipudi

Sawyer is the principal investigator for the Louisiana Tech group and is in charge of grant administration. He has an extensive background in calorimetry, including the design and construction of the $\mathrm{D} \varnothing$ Intercryostat Detector, early design and testbeam work on the ATLAS tile calorimeter, and design studies for calorimetry in several experiment, which include experiments for the proposed International Linear Collider. Sawyer's service work on the ATLAS experiment has been centered around Data Quality Monitoring of the combined calorimeter monitoring (referred to as "CaloGlobal" or by the name of the software package "CaloMonitoring"). Currently, he and his student Khadeejah Alghadeer are responsible for maintenance of much of the CaloMonitoring tools. Alghadeer is the on-call expert at CERN for CaloGlobal, taking over from a Columbia University student in August 2012.

Sawyer's physics research work is largely involved with multijet measurements and QCD studies. Working in close collaboration with Wobisch, Tamsett, and the students in the Louisiana Tech jet physics subgroup, he has been the lead analyzer on several of the group's recent analyses described below.

\subsection{Calorimeter Data Quality Monitoring}

The ATLAS calorimeter system is a multi-component collection of devices including lead/liquid Argon-based (LAr) calorimeters with accordion geometry for the central and endcap electromagnetic calorimeter, presamplers, and hadronic endcap; iron/scintillating tile-based calorimeters for the barrel and extended barrel hadronic calorimetry, and a specialized copper/tungsten/LAr 
forward calorimeter. Both online and offline Data Quality (DQ) Monitoring play an important role in the operation and effectiveness of the ATLAS detector for physics studies. CaloMonitoring is one of the tools used in achieving this task. The starting point is the measurement of the energy deposited in cells of the calorimeters. Extended objects such as clusters and towers are built by grouping the cells in a predefined logic. The information on cells, clusters, and towers is stored as containers in an ESD (Event Summary Data). A DQ assessment is made using the CaloMonitoring tools on these containers and reported as a flag in a database for each run. The CaloMonitoring package includes monitoring algorithms based on clusters, towers, and calorimeter cells.

The CaloMonitoring package itself is a collection of tools to produce histograms of occupancy, average energy, and hit maps of dominant cell and average number of cells in the clusters or towers. Several kinds of calorimeter objects are produced (CaloCalTopoCluster, EMTopoCluster and CombinedTower), and monitoring histograms can be selected for each of these. These histograms are used to look for hot regions which show up as peaks in the distributions in pseudorapidity and azimuthal angle. Both Sawyer and the students have written much of this code and have maintained this software package.

The histograms are produced for the full detector coverage of -5.0 to +5.0 in pseudorapidity and $-\pi$ to $+\pi$ in azimuthal angle. The histograms can be examined by eye for any peaks, or an intelligent algorithm which employs statistical methods can be applied. We make use of an algorithm to look for regions deviating from the mean in the determination of the problematic regions. The ATLAS Data Preparation group provides a DQWebDisplay tool to be used as a common platform to display the histograms and DQ assessment on the web for all the systems assessing the DQ for the detector. The problems reported by various systems in the daily DQ meetings are analyzed to see any correspondence in the problems spotted by related systems. Necessary corrective steps are taken to propagate the information to the responsible personnel and the data is reprocessed for final use by all users for physics analysis.

Sawyer supervises student Alghadeer in her duties as CaloGlobal expert, which include making the DQ assessment on the data and reporting the efficiency of the automatic tests configured through the DQWebDisplay. Further responsibilities include filling the CaloGlobal flags, reporting the assessment in the daily DQ meetings, and reporting the results of the assessment within the CaloGlobal group. Alghdeer also maintains the configuration that drives the DQ assessment using the DQWebDisplay.

In addition to software maintenance, both Sawyer and Alghadeer, as well as graduate student Sircar, regularly take offline CaloGlobal data quality shifts. In addition, Sawyer takes shifts at the DQ desk in the ATLAS control room. Responsibilities include inspecting the proper functioning of the monitoring infrastructure and reporting to the corresponding experts to fix them, looking at problems in different systems, determining the cause of the problem by communicating with other shifters at point 1 and reporting them in a daily elogs. These tasks are part of the overall data preparation.

During fall 2012, the CaloGlobal offline shifts were merged with calorimeter-based Combined Performance (CP) shifts, specifically jet/Etmiss, tau, e/gamma offline shifts. Shifter duties include looking at jet physics quantities of interest; determining the DQ of the jets, missing $E_{T}$, electrons, photons, and taus; using the diagnostic tools; and reporting the problems and DQ assessment for the runs taken during a 24 hour period; and correlating the problems with the 
problems seen at the detector level. Sawyer and Alghadeer took leading roles in the development and implementation of these Combined Calorimeter CP shifts, with Alghdeer serving as the Data Quality group CaloGlobal contact. The Louisiana Tech group, cited as one of the largest and most experienced groups by the data quality shift organizers, piloted the first of these combined calorimeter shifts during the second week of September 2012, and was the only US group contributing to these shifts during the remainder of the 2012-2013 run.

\subsection{Jet Trigger Upgrades}

As mentioned in Wobisch's section, the Louisiana Tech group has become very active in the ATLAS jet trigger signature group, playing a leading role in the development of the Level 1.5 jet trigger. Sawyer is part of this effort, and in particular, has been studying the implementation of various jet finding algorithms at Level 2. This work intended both for the current running and as a study for possible upgrades to the Level 1 jet trigger.

\section{Other Jet Trigger Activities}

In addition to upgrade studies, Sawyer has helped with the L1.5 jet energy calibration. His student Alghadeer is working on jet trigger monitoring in addition to her work in the CaloGlobal data quality effort. It is envisioned that during the shutdown in 2014, the jet trigger monitoring will be a large part of her service work and a major portion of her authorship task.

\subsection{Jet Physics at DØ and ATLAS}

One of the strengths of the Louisiana Tech High Energy Physics group is its expertise in the area of jet physics studies at hadron colliders. This work is the outgrowth of the close collaboration among Sawyer, Wobisch, their postdocs, and their students. Sawyer was the lead analyzer of the measurement of multijet ratios, $R_{3 / 2}$ at $\mathrm{D} \emptyset$ and lead author of the paper which was recently submitted to Physics Letters B. He supervised Scott Atkins in his dissertation research on transverse jet event shapes at $\mathrm{D} \emptyset$ which will evolve into a paper later this fall. Sawyer also supervised Ram Dhullipudi on his dissertation study of the $p_{T}$-dependence of the dijet decorrelations using the 2010 data from the ATLAS experiment. This work is currently being updated for the 2012 data by Tamsett, Sawyer, and Wobisch. Sawyer's current student on ATLAS, Alghadeer, is scheduled to work on three-jet and four-jet cross sections at ATLAS using the 2012 data, for which Sawyer has written the preliminary version of the data analysis software.

\section{Multijet Ratios}

As discussed previously in this proposal, Louisiana Tech is pioneering several novel analyses based on ratios of multijet cross sections. In section 2 we discussed the three multijet ratios, $R_{3 / 2}, R_{\Delta \phi}$, and $R_{\Delta R}$. Each of these have been developed on the $\mathrm{D} \emptyset$ experiment and are now being studied in the ATLAS data. As most of these analyses have been defined in section 2 and discussed in some detail in section 4, only a few specifics of Sawyer's contributions will be mentioned here.

Observables which are insensitive to PDFs include conditional probabilities for a certain type of event topology, given that another event topology was already observed. The conditional 


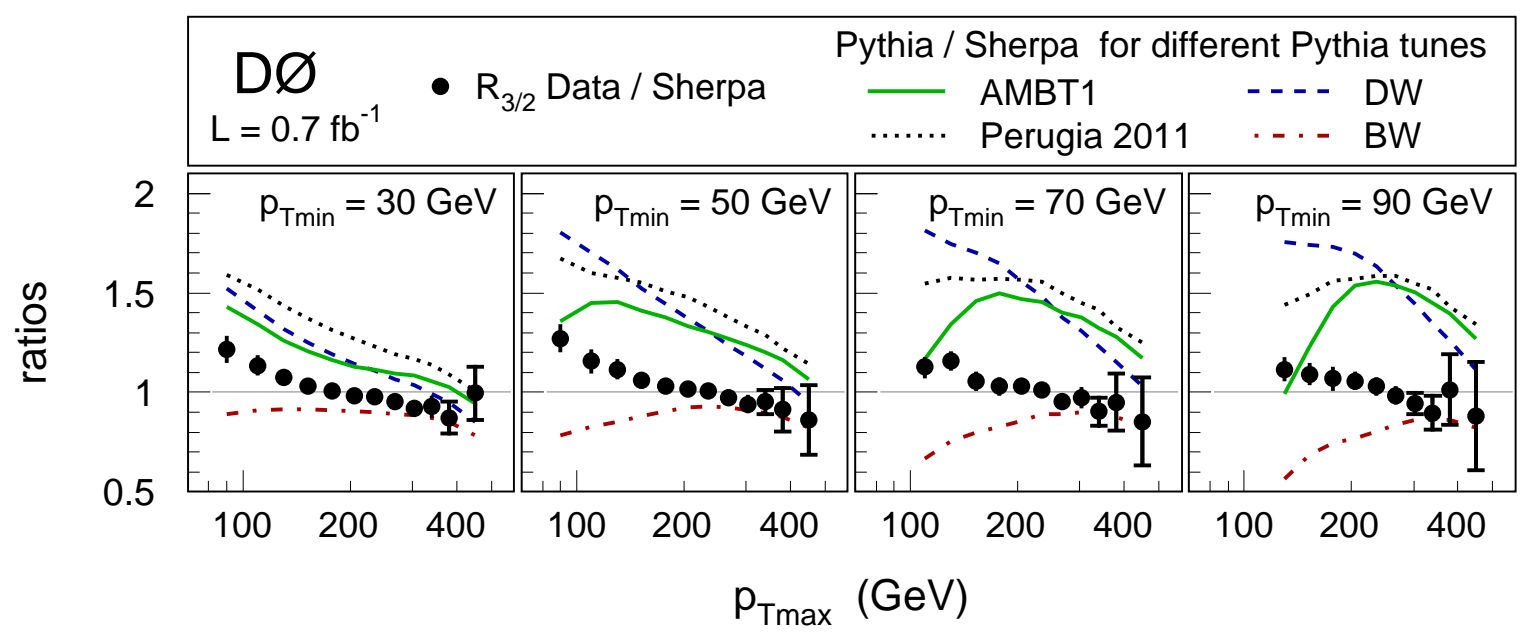

Figure 3: The measured $R_{3 / 2}$ results, normalized to the predictions of the SHERPA Monte Carlo event generator. The inner uncertainty bars represent the statistical uncertainties while the total uncertainty bars represent the quadratic sums of statistical and systematic uncertainties. Overlaid are the predictions from the PYTHIA Monte Carlo event generator for four different tunes, also normalized to the SHERPA predictions.

probability of finding a third jet in an event in which two jets have already been observed is called $R_{3 / 2}$. Technically this is the ratio of the inclusive three-jet cross section and the inclusive dijet cross section. In our analyses, $R_{3 / 2}$ is measured as a function of leading jet $p_{T}\left(p_{T \text { max }}\right)$ for different $p_{T}^{\min }$ requirements (where $p_{T}^{\text {min }}$ is the $p_{T}$ of the $n$-th jet in the $n$-jet event sample). Our $\mathrm{D} \emptyset$ results for this measurement (obtained by Atkins, Sawyer, Wobisch) have been submitted to Physics Letters B. The results are compared to the NLO pQCD theory predictions in Fig. 1 (bottom left), which agree well with the data. Predictions from different Monte Carlo event generators are also compared to the data, as shown in Fig. 3. In this figure, the measured $R_{3 / 2}$ results and the PYTHIA predictions for different tunes are divided by the predictions from SHERPA which includes the tree-level matrix elements for two-, three-, and four-jet production, matched with a parton shower. The SHERPA predictions for $R_{3 / 2}$ agree with the data within $-20 \%$ to $+10 \%$. The PYTHIA predictions depend strongly on the parameter settings used, and none of the tunes investigated here agree with the data.

This analysis has also been made by Tamsett and Wobisch using the ATLAS 2011 data at $\sqrt{s}=7 \mathrm{TeV}$. The result was included in an ATLAS multi-jets paper [13] during the past year.

Sawyer was the lead analyzer and author of the paper on the 3 -jet to 2 -jet ration, $R_{3 / 2}$, at $\mathrm{D} \emptyset$, which has been submitted to Physics Letters B. This measurement is used to exclude various tunes of the PYTHIA event generator, and compared to the NLO pQCD theory predictions which agree well with the data, as shown in Fig. 1 (bottom left). He also wrote the data analysis software used for the $\mathrm{D} \emptyset R_{\Delta \phi}$ measurement (Fig. 1, top right)and was co-author with Wobisch on the paper on $R_{\Delta R}$ submitted to Physics Letters $\mathrm{B}$.

On ATLAS, Dhullipudi and Sawyer performed the first $R_{\Delta \phi}$ measurement at the LHC, using the initial 2010 dataset at $\sqrt{s}=7 \mathrm{TeV}$, with Sawyer performing the calculations of the non- 
perturbative corrections using the RIVET Monte Carlo interface, as well as supervising Dhullipudi's analysis of the data. This analysis was the basis of Dhullipudi's dissertation. Currently, Sawyer, Wobisch, and Tamsett are extending the analysis to the 2012 ATLAS dataset at $\sqrt{s}=8 \mathrm{TeV}$.

\section{Multijet Cross Sections}

Multijet cross sections, differential in the invariant mass of the multijet system, are one of the classical observables to test theory predictions. These observables can be used to search for physics beyond the standard model which either manifests itself in monotonous deviations at high masses (signaling the presence of new physics at higher scales modeled by contact interactions) or in resonant structures (indicating the presence of new particles, decaying to multi-jets).

Previous measurements of inclusive jet and dijet production in $p \bar{p}$ collisions have demonstrated the success of pQCD in describing observables which are directly sensitive to the matrix elements of order $\alpha_{s}^{2}$. Testing pQCD at higher orders of $\alpha_{s}$ requires to measure cross sections for higher jet multiplicities. Together with Z. Hubacek (University Prague), Wobisch has made a first measurement of the three-jet cross section in $p \bar{p}$ collisions at $\sqrt{s}=1.96 \mathrm{TeV}$ [3]. For the ATLAS experiment, Sawyer is performing the measurement of the three-jet cross section using the 2012 data set. A preliminary version of the analysis is planned to be presented within the collaboration in fall 2012. Eventually this will form part of Alghadeer's dissertation, which may also include the four-jet cross section measurement. The observable is the differential three-jet cross section as a function of the three-jet invariant mass (for different rapidity and $p_{T}$ requirements) which is directly sensitive to the pQCD matrix elements of order $\alpha_{s}^{3}$. 


\section{Bibliography and References Cited}

[1] V. M. Abazov et al. [D0 Collaboration], "Measurement of dijet angular distributions at sqrt(s) $=1.96 \mathrm{TeV}$ and searches for quark compositeness and extra spatial dimensions," Phys. Rev. Lett. 103, 191803 (2009) [arXiv:0906.4819 [hep-ex]].

[2] V. M. Abazov et al. [D0 Collaboration], "Measurement of the dijet invariant mass cross section in proton anti-proton collisions at sqrts = 1.96 TeV," Phys. Lett. B 693, 531 (2010) [arXiv:1002.4594 [hep-ex]].

[3] V. M. Abazov et al. [D0 Collaboration], "Measurement of three-jet differential cross sections $d \sigma_{3 \mathrm{jet}} / d M_{3 \mathrm{jet}}$ in $p \bar{p}$ collisions at $\sqrt{s}=1.96 \mathrm{TeV}$," Phys. Lett. B 704, 434 (2011) [arXiv:1104.1986 [hep-ex]].

[4] V. M. Abazov et al. [D0 Collaboration], "Determination of the strong coupling constant from the inclusive jet cross section in ppbar collisions at sqrt(s) $=1.96 \mathrm{TeV}$," Phys. Rev. D 80, 111107 (2009) [arXiv:0911.2710 [hep-ex]].

[5] V. M. Abazov et al. [D0 Collaboration], "Measurement of angular correlations of jets at $\sqrt{s}=1.96 \mathrm{TeV}$ and determination of the strong coupling at high momentum transfers," Phys. Lett. B 718, 56 (2012) [arXiv:1207.4957 [hep-ex]].

[6] M. Wobisch, K. Chakravarthula, R. Dhullipudi, L. Sawyer and M. Tamsett, "A new quantity for studies of dijet azimuthal decorrelations," JHEP 1301, 172 (2013) [arXiv:1211.6773 [hep-ph]].

[7] V. M. Abazov et al. [D0 Collaboration], "Measurement of the ratio of three-jet to two-jet cross sections in $p \bar{p}$ collisions at $\sqrt{s}=1.96$ TeV," Phys. Lett. B [Phys. Lett. B 720, 6 (2013)] [arXiv:1209.1140 [hep-ex]].

[8] V. M. Abazov et al. [ D0 Collaboration], "Measurement of the combined rapidity and $p_{T}$ dependence of dijet azimuthal decorrelations in $p \bar{p}$ collisions at $\sqrt{s}=1.96 \mathrm{TeV}$," arXiv:1212.1842 [hep-ex].

[9] G. Aad et al. [ATLAS Collaboration], "Measurement of the top quark pair cross section with ATLAS in pp collisions at sqrt(s) $=7 \mathrm{TeV}$ using final states with an electron or a muon and a hadronically decaying $\tau$ lepton," arXiv:1205.2067 [hep-ex].

[10] G. Aad et al. [ATLAS Collaboration], "Search for charged Higgs bosons decaying via H+ -i tau nu in top quark pair events using pp collision data at sqrt(s) $=7 \mathrm{TeV}$ with the ATLAS detector," JHEP 1206, 039 (2012) [arXiv:1204.2760 [hep-ex]].

[11] P. Assamagan, C. Bernius, P Casado, V. Cavaliere, P. Czodrowski, D. Chakraborty, D. Greenwood, A. Ferrari, M. Flechl, S. Hattrem Raddum, A. Kopp, P. Mal, A. McCarn, M. Neubauer, N. Patel, A. Randle-Conde, A. L. Read, G Rocha de Lima, A. Saavedra, M. Schumacher, S. Sekula, "Search for charged Higgs bosons in the tau+jets final state in ttbar decays with $4.6 \mathrm{fb}-1$ of pp collision data recorded at sqrt(s) $=7 \mathrm{TeV}$ with the ATLAS Experiment", ATL-PHYS-INT-2012-047 (2012) 
[12] K Nakamura et al. (Particle Data Group), J. Phys. G 37 , 7A, 075021 (2010).

[13] G. Aad et al. [ATLAS Collaboration], "Measurement of multi-jet cross sections in protonproton collisions at a 7 TeV center-of-mass energy," Eur. Phys. J. C 71, 1763 (2011), arXiv:1107.2092 [hep-ex].

[14] T. Kluge, K. Rabbertz and M. Wobisch, "FastNLO: Fast pQCD calculations for PDF fits," hep-ph/0609285.

[15] M. Wobisch et al. [fastNLO Collaboration], "Theory-Data Comparisons for Jet Measurements in Hadron-Induced Processes," arXiv:1109.1310 [hep-ph].

[16] A. D. Martin, W. J. Stirling, R. S. Thorne and G. Watt, "Parton distributions for the LHC," Eur. Phys. J. C 63, 189 (2009).

[17] A. D. Martin, W. J. Stirling, R. S. Thorne and G. Watt, "Uncertainties on $\alpha_{s}$ in global PDF analyses," arXiv:0905.3531 [hep-ph].

[18] H. L. Lai et al., "New parton distributions for collider physics," Phys. Rev. D 82, 074024 (2010).

[19] M. Gluck, P. Jimenez-Delgado and E. Reya, "Dynamical parton distributions of the nucleon and very small-x physics," Eur. Phys. J. C 53, 355 (2008) [arXiv:0709.0614 [hep-ph]].

[20] A. Vogt, "Parton Distributions: Progress and Challenges," arXiv:0707.4106 [hep-ph].

[21] D. de Florian, R. Sassot, M. Stratmann and W. Vogelsang, "Extraction of SpinDependent Parton Densities and Their Uncertainties," Phys. Rev. D 80, 034030 (2009) [arXiv:0904.3821 [hep-ph]].

[22] D. Britzger et al. [fastNLO Collaboration], "New features in version 2 of the fastNLO project," arXiv:1208.3641 [hep-ph]. 